\title{
Dynamic Contrast-Enhanced Magnetic Resonance Imaging as a Surrogate Biomarker for Bevacizumab in Colorectal Cancer Liver Metastasis: A Single-Arm, Exploratory Trial
}

\author{
Yeo-Eun Kim, MD ${ }^{1,2}$ \\ Bio Joo, MD2 \\ Mi-Suk Park, MD2 \\ Sang Joon Shin, $\mathrm{MD}^{3}$ \\ Joong Bae Ahn, MD³ \\ Myeong-Jin Kim, MD2
}

\begin{abstract}
${ }^{1}$ Department of Diagnostic Radiology, Seoul Medical Center, Seoul,

${ }^{2}$ Department of Diagnostic Radiology, Research Institute of Radiological Science, Severance Hospital, Yonsei University

College of Medicine, Seoul,

${ }^{3}$ Division of Medical Oncology, Department of Internal Medicine, Yonsei Cancer Center, Yonsei University College of Medicine, Seoul, Korea
\end{abstract}

Correspondence: Mi-Suk Park, MD Department of Diagnostic Radiology, Research Institute of Radiological Science, Severance Hospital, Yonsei University College of Medicine, 50-1 Yonsei-ro, Seodaemun-gu, Seoul 03722, Korea Tel: 82-2-2228-7400

Fax: 82-2-393-3035

E-mail: radpms@yuhs.ac

Co-correspondence: Sang Joon Shin, MD Division of Medical Oncology, Department of Internal Medicine, Yonsei Cancer Center, Yonsei University College of Medicine, 50-1 Yonsei-ro, Seodaemun-gu, Seoul 03722, Korea Tel: $82-2-2228-8138$

Fax: 82-2-393-3652

E-mail: SSJ338@yuhs.ac

Received October 4, 2015

Accepted March 8, 2016

Published Online March 17, 2016

*Yeo-Eun Kim and Bio Joo contributed equally to this work.

\section{Purpose}

The purpose of this study is to investigate dynamic contrast-enhanced magnetic resonance imaging (DCE-MRI) and plasma cytokines and angiogenic factors (CAFs) as pharmacodynamic and prognostic biomarkers of bevacizumab monotherapy in colorectal cancer with liver metastasis (CRCLM).

\section{Materials and Methods}

From July 2011 to March 2012, 28 patients with histologically confirmed CRCLM received bevacizumab monotherapy followed by combined FOLFOX therapy. The mean age of the patients was 57 years (range, 30 to 77 years). DCE-MRI (Krans and IAUC60) was performed at baseline, first follow-up (3 days after bevacizumab monotherapy), and second follow-up (3 days after combined therapy). CAF levels (vascular endothelial growth factor [VEGF], placental growth factor [PIGF], and interleukin-8) were assessed on the same days. Progression-free survival (PFS) time distributions were summarized using the Kaplan-Meier method and compared using log-rank tests.

\section{Results}

The median PFS period was 11.2 months. $K^{\text {trans }}$, IAUC60, VEGF, and PIGF values on the first follow-up day were significantly different compared with baseline values. No differences were observed on the second follow-up day. $A>40 \%$ decrease in $\mathrm{K}^{\text {trans }}$ from baseline to first follow-up was associated with a longer PFS (hazard ratio, 0.349; 95\% confidence interval, 0.133 to $0.912 ; p=0.032$ ). Changes in CAFs did not show correlation with PFS time.

\section{Conclusion}

DCE-MRI parameters and CAFs are pharmacodynamic biomarkers of bevacizumab for CRCLM. In our study, change in $\mathrm{K}^{\text {trans }}$ at 3 days after bevacizumab monotherapy was a favorable prognostic factor; however, the value of CAFs as a prognostic biomarker was not found.
Key words

Dynamic contrast enhanced-magnetic resonance imaging, Bevacizumab, Colorectal neoplasms 


\section{Introduction}

The combination of bevacizumab with cytotoxic chemotherapy has been proved to have survival benefits in patients with metastatic colorectal cancer [1-3]. This combination has been approved by the US Food and Drug Administration (Washington, DC) as a first-line treatment [2]. Bevacizumab is an inhibitor of vascular endothelial growth factor (VEGF). It normalizes the abnormal vascular structures in tumor stroma, decreases the elevated interstitial pressure in tumors, and alleviates the hypoxic tumor microenvironment [4]. In addition to the direct antiangiogenic effects, these changes also increase chemotherapeutic efficacy. The standard protocol uses combination therapy with cytotoxic agents but does not include bevacizumab mono-therapy [2]. One major issue for the combination therapy is that clinicians cannot distinguish the effects of bevacizumab alone from the effects of the cytotoxic agents used in the protocol. Despite the benefit of bevacizumab on clinical outcome, a substantial number of patients do not respond to the therapy targeting VEGF, or they develop resistance to the drug over time. There is also an increased chance of serious side effects (e.g., stroke and gastrointestinal perforation) in patients treated with VEGF-targeted therapy $[2,5]$. In addition, the target agents are expensive. There is a critical need for identification of reliable biomarkers for prediction of tumor response to treatment and to predict which patients are likely to benefit from bevacizumab.

Plasma cytokines and angiogenic factors (CAFs) are potential biomarkers that reflect the systemic condition of patients with colorectal cancer treated with bevacizumab combined with cytotoxic chemotherapy [6]. Elevated baseline interleukin 8 (IL-8) is associated with a shorter progression-free survival (PFS) time. Levels of some plasma CAFs associated with angiogenesis and myeloid recruitment increase compared to baseline, before radiographic progression [7]. Plasma CAFs could be potential biomarkers of response and resistance to bevacizumab, but they cannot reflect the local response of the tumor microenvironment and cannot differentiate the response of the tumor from that of the host tissue [7].

Unlike plasma CAFs used as systemic biomarkers, an imaging biomarker can reflect local tumor condition. However, tumor size may not be a reliable marker for assessment of the early effects of antiangiogenic agents because many of these new drugs act directly on endothelial cells, not on the tumor cells. Therefore, the effects of the agents are expected to be primarily cytostatic rather than cytotoxic [8]. As an alternative functional modality, dynamic contrast-enhanced magnetic resonance imaging (DCE-MRI) is a noninvasive and appropriate method for evaluating the efficacy of these antiangiogenic therapies using changes in tumor vascular perfusion, vascular permeability, and interstitial and intravascular volumes, without exposure to ionizing radiation [8]. The volume transfer constant $\left(\mathrm{K}^{\text {trans }}\right)$ and the initial area under the gadolinium concentration-time curve (IAUC) have been proposed as useful parameters that reflect the vascular response [8]. These parameters can be used with DCE-MRI to quantify the effects of antiangiogenic therapy during the early response period.

Several studies have reported reduction in $\mathrm{K}^{\text {trans }}$ within a few days of bevacizumab treatment $[8,9]$. Some studies of bevacizumab in colorectal cancer suggest that DCE-MRI may be related to clinical outcome $[10,11]$. In addition, several studies of anti-VEGF tyrosine kinase inhibitors have shown that reduction in $\mathrm{K}^{\mathrm{K} \text { tans }}$ and / or IAUC may distinguish responders from non-responders [12-14]. Based on those studies, patients with a smaller change in $\mathrm{K}^{\text {trans }}$ are predicted to have a shorter PFS time and to receive less clinical benefit from the treatment. However, when applying the results in the clinical practice setting, the clinician must determine whether the patients should stop receiving only bevacizumab, only the cytotoxic agent, or both bevacizumab and the cytotoxic agent. This is because the clinician cannot determine whether the change in $\mathrm{K}^{\text {trans }}$ results from the effect of the bevacizumab alone, the cytotoxic agent, or both.

To gain insight into the mechanisms of action and the pure effects of bevacizumab, we designed an exploratory trial with a single-arm of bevacizumab monotherapy followed by combined therapy. The effects of bevacizumab alone on both tumor physiology and systemic response were evaluated by measuring DCE-MRI parameters and plasma CAF levels. The primary objective of this phase II clinical trial was to determine prognostic biomarkers of bevacizumab alone that might show correlation with clinical outcomes. Our study also revealed the antiangiogenic effect of bevacizumab monotherapy on local tumor response using changes in DCE-MRI, and on systemic response using changes in plasma CAFs.

\section{Materials and Methods}

This study was registered at http://www.clnicaltrials.gov (NCT01318239). The study was conducted in accordance with the Declaration of Helsinki and Good Clinical Practice Guidelines. Protocol approval was obtained from the Institutional Review Board of Severance Hospital, Yonsei University College of Medicine (Seoul, Korea). Written informed consent was obtained from each patient. 


\section{Patient population}

Patients aged $\geq 20$ years with histologically confirmed colorectal cancer and one or more measurable hepatic metastases $\geq 2 \mathrm{~cm}$ diameter were enrolled in the study.

Patients with standard contraindications for MRI and/or gadolinium-based contrast agents, pregnant or breast-feeding women, and patients with decreased renal function (creatinine $>1.5 \mathrm{mg} / \mathrm{dL}$, blood urea nitrogen $>30 \mathrm{mg} / \mathrm{dL}$ ), who had prior chemotherapy, concurrent malignancies or significant medical comorbidities, and who had received any treatment as part of a clinical trial were excluded from the study.

\section{Study design}

In this single group, exploratory trial, patients were treated with bevacizumab (5 $\mathrm{mg} / \mathrm{kg}$ intravenous) alone on day 1 , followed by a concurrent FOLFOX-6+B regimen (bevacizumab/oxaliplatin/5-fluorouracil/ leucovorin) on day 7 . Reduction in dose of the cytotoxic chemotherapy was required for grade 3 or 4 toxicities that were adverse events of however, the dose of bevacizumab, was not reduced. Adverse events were graded using the National Cancer Institute Common Terminology Criteria for Adverse Events ver. 3.0. Treatment was continued until documentation of unacceptable toxicities, disease progression, or withdrawal of consent.

DCE-MRI and blood sampling for plasma CAFs were performed on the day before treatment was initiated (baseline), on day 3 after the patients received bevacizumab monotherapy (first follow-up), and on day 3 after receiving the combined chemotherapy (second follow-up) (Fig. 1).

The primary endpoint was the correlation between the changes in DCE-MRI parameters (from baseline to 3 days after administration of bevacizumab monotherapy) with PFS time. The standard DCE-MRI parameters ( $\mathrm{K}^{\text {trans }}$ and IAUC $\left.{ }_{60}\right)$ were measured during each imaging and the changes from baseline to first follow-up and from first follow-up to second follow-up were calculated. The baseline values were dichotomized according to the low and high group based on the median value. The changes in the DCE-MRI parameters were dichotomized according to larger and smaller change groups, using a $40 \%$ change as the cut-off value. This cut-off value of $40 \%$ was chosen because a $40 \%$ reduction in $\mathrm{K}^{\text {trans }}$ is necessary before a favorable clinical response can be detected $[11-13,15,16]$. Dichotomized values of the baseline and the changes were correlated with PFS. The changes in tumor size were also measured at baseline, first follow-up, and second follow-up.

The secondary endpoints included the correlation between changes in plasma CAFs from baseline to 3 days after beva- cizumab monotherapy, with PFS and toxicity. For correlation with clinical outcomes, plasma CAF values were dichotomized by calculating optimal cut-off values, obtained from receiver operating characteristic curve analysis.

PFS was defined as the period from the enrollment date until the documented tumor progression date. Data were censored for the patients who were alive without disease progression at the last follow-up date. Computed tomography (CT) examination with contrast enhancement was performed before and every 3 months after treatment. Tumor response evaluated at 3 months after the treatment was dichotomized according to a partial response group and a stable disease or disease progression group. Assessment of tumor response was performed in accordance with the Response Evaluation Criteria in Solid Tumors (RECIST) ver. 1.1 guidelines [17].

\section{DCE-MRI study}

For reproducibility, consistent DCE-MRI methods were followed using a 3-T MR scanner (MAGNETOM Tim Trio, Siemens Healthcare, Erlangen, Germany) equipped with 8-channel body phased-array coils (Siemens Healthcare). Each patient fasted for at least 4 hours before the scan was performed.

$\mathrm{T}_{1}$ mapping was obtained before acquisition of perfusion images to convert signal intensities of perfusion images into gadolinium concentration values. $T_{1}$ maps were calculated from two flip angle scans using $2^{\circ}$ and $14^{\circ}$ :

$$
\mathrm{S}(\alpha, T 1, T R)=\mathrm{S}_{0} \frac{\operatorname{Sin} \alpha(1-\exp (-T R / T 1))}{1-\cos (\alpha) \exp (-T R / T 1)}, T_{1}(t)=T_{10}+\mathrm{r}_{1}{ }^{*} \mathrm{c}(t)
$$

where $T_{1}(t)$ : $T 1$ dynamic; $T_{10}$ : $T 1$ before contrast; $r_{1}: T_{1}$ relaxivity of the contrast agent; and $c(t)$ : contrast agent concentration.

Axial images for the T1 maps were obtained using threedimensional (3D) spoiled gradient echo sequences with two different flip angles $\left(2^{\circ}\right.$ and $\left.14^{\circ}\right)$ and a fixed repetition time/echo time (TR/TE; $4.91 \mathrm{msec} / 1.71 \mathrm{msec}$ ). A total of 20 slices per sequence using a different flip angle were acquired. The slice thickness was $3.6 \mathrm{~mm}$ and the in-plane resolution was $1.67 \times 1.67 \mathrm{~mm}^{2}$ for each in one breath-hold and scan time of 18 seconds.

Perfusion images were obtained using 3D T1-weighted time resolved angiography with stochastic trajectories (TWIST) sequence (TR/TE, $4.51 \mathrm{msec} / 1.76 \mathrm{msec}$; slice thickness, $3.6 \mathrm{~mm}$; flip angle, $12^{\circ}$; matrix size, $192 \times 138$; field of view, $300 \mathrm{~mm}$; band width, $250 \mathrm{~Hz} / \mathrm{Px}$ ). Serial images (temporal resolution, 0.295 seconds) were acquired during shallow, free-breathing respiration. Twenty axial image slices 


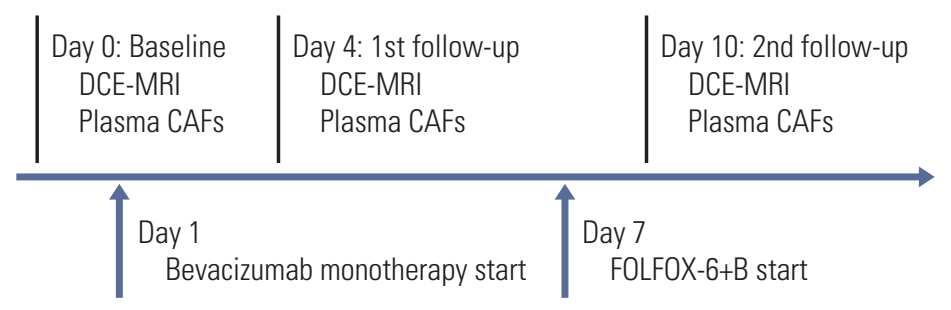

Fig. 1. Study design. Dynamic contrast-enhanced magnetic resonance imaging (DCE-MRI) studies and blood sampling for plasma cytokine and angiogenic factors (CAFs) were performed 1 day before treatment (day 0 ). Patients were treated with bevacizumab ( $5 \mathrm{mg} / \mathrm{kg}$ intravenously) alone on day 1 and the first follow-up DCE-MRI and blood sampling for plasma CAFs were performed 3 days after the monotherapy treatment (day 4). A concurrent FOLFOX-6+B regimen (bevacizumab / oxaliplatin/5-fluorouracil/leucovorin) was administered on day 7 and the second follow-up DCE-MRI and blood sampling for plasma CAFs were performed 3 days after combined chemotherapy (day 10).

(range, $10 \mathrm{~cm}$ ) per cycle were obtained while centered on the center of the tumor (5.9 seconds for one cycle). Perfusion images were acquired repetitively over 75 cycles for 7-8 minutes. A total volume of $15 \mathrm{~mL}$ Omniscan (gadodiamide, GE Healthcare, Oslo, Norway) was used as a contrast material, and was injected intravenously using an automatic injector at a rate of $5 \mathrm{~mL} / \mathrm{sec}$, followed by a $30-\mathrm{mL}$ saline injection. An algorithm with a diffeomorphism flow was introduced for registration between the T1 mapping sequence and the perfusion imaging sequence. The algorithm was based on maximization of statistical similarity criteria (local and global) in a variational framework. This flow was adjusted using a template propagation method by composition of small displacements. Regularization was achieved using fast filtering techniques. This approach combined the robustness of statistical similarity measures with the flexibility of diffeomorphic matching techniques [18].

A commercial post-processing software based on a modified Tofts model (Tissue 4D, Siemens Medical Solutions) and installed at an image processing workstation (Leonardo, Siemens Medical Solutions), was used for calculation of the quantitative perfusion parameters. Motion correction was performed using the non-rigid body algorithm on the postprocessing software. For analysis of the perfusion data, the arterial input function was set as the average bi-exponential function using fast mode. Color-coded maps of the $\mathrm{K}^{\text {trans }}$ values were acquired through data processing. IAUC 60 values for the first 60 s from the contrast injection were also acquired. Two dimensional regions of interest (ROIs) were handdrawn on post-processed images by an abdominal radiologist with 7 years experience, according to the tumor margin through the midline of the z-axis of the tumor in the section where the largest transverse diameter of liver metastasis was detected. ROIs were drawn for all tumors $>2 \mathrm{~cm}$ in diameter and a mean value for $\mathrm{K}^{\text {trans }}$ was estimated for all patients with multiple lesions. Unlike RECIST, the commonly used criterion for measurable lesions on DCE-MRI is $>2 \mathrm{~cm}$ in diameter, in consideration both of size reduction and measurement error [10].

The baseline $\mathrm{K}^{\text {trans }}$ and $\mathrm{IAUC}_{60}$ values were acquired from the baseline study results. The values for these same parameters were assessed from the first follow-up and second follow-up images. The changes were calculated as absolute and percentage values.

\section{Plasma sample collection and analysis}

Blood samples were collected for circulating pharmacodynamics marker studies prior to the initial bevacizumab infusion, on the third day after bevacizumab monotherapy, and on the third day after combined therapy. Serum samples were assayed for circulating VEGF-A, placental growth factor (PIGF), soluble vascular endothelial growth factor receptor 2 (sVEGFR2), and IL-8 using enzyme-linked immunosorbent assay kits (R\&D Systems, Minneapolis, MN). The changes were calculated as absolute and percentage values.

\section{Statistical analysis}

DCE-MRI parameter and plasma CAF values were correlated with PFS. Baseline values and the changes in the DCEMRI parameters and CAF values were analyzed to determine correlations among the parameters.

Kaplan-Meier estimates and log-rank tests were computed to evaluate the associations between changes in DCE-MRI parameter or plasma CAF values with the length of PFS or overall survival (OS). Analyses of associations between baseline values of DCE-MRI parameters or plasma CAFs with PFS or OS were also performed.

The PFS and OS times were estimated using the Kaplan- 
Meier method. The differences among the patient groups for PFS, OS, and treatment response were compared using multivariate Cox regression analysis. Six parameters were considered as possible covariates in the regression analysis. The associations among the categorical variables and the continuous variables were analyzed using chi-square tests and linear mixed models, respectively.

For all analyses, a p-value $<0.05$ was considered statistically significant. SPSS ver. 18.0 (SPSS Inc., Chicago, IL) and SAS ver. 9.2 (SAS Institute Inc., Cary, NC) were used for all analyses.

\section{Results}

\section{Patient characteristics and clinical outcomes}

Thirty-two patients were enrolled in the study between July 2011 and March 2012. Four patients were excluded from the analysis due to follow-up loss $(n=3)$ or imaging error $(\mathrm{n}=1)$. The mean age of the patients was 57 years (range, 30 to 77 years), and a total of 60 hepatic metastatic lesions in patients with rectal cancer $(n=8)$ and colon cancer $(n=20)$ were analyzed. The Eastern Cooperative Oncology Group performance status score was 0 for 22 patients and 1 for six patients. The mean baseline carcinoembryonic antigen value was $340.78 \mathrm{ng} / \mathrm{mL}$.

The median PFS time was 11.2 months (95\% confidence interval [CI], 6.9 to 15.5), and the median OS time was 22.7 months (95\% CI, 18.8 to 26.7).

In assessment of tumor response on 3-month follow-up CT images according to RECIST ver. 1.1, 13 patients showed partial response and 14 patients showed stable disease. Only one patient showed disease progression on the 3-month followup. Dose reduction was required for five of 28 patients due to toxicity during the course of the scheduled treatment. Fatigue and neuropathy were the most common adverse events. Neutropenia was the most frequent of the grade 3 or 4 adverse events (Table 1 ).

\section{DCE-MRI parameters and plasma CAFs as pharmacody- namic biomarkers}

The results for the changes in DCE-MRI parameters, plasma CAF values, and tumor size at 3 days after bevacizumab monotherapy (first follow-up) and at 3 days after combined chemotherapy (second follow-up) are shown in Fig. 2. Compared with the baseline values, the $\mathrm{K}^{\text {trans }}$, IAUC ${ }_{60}$, and serum VEGF values at the first follow-up evaluation had decreased $(p<0.05)$. No significant changes were detected
Table 1. Adverse events after treatment

\begin{tabular}{|c|c|c|}
\hline \multirow{2}{*}{ Event } & \multicolumn{2}{|c|}{ No. of patients $(n=28)$} \\
\hline & Grade 3 or 4 & All grades \\
\hline Neutropenia & 8 & 10 \\
\hline Nausea & 4 & 13 \\
\hline Vomiting & 1 & 2 \\
\hline Ileus & 3 & 4 \\
\hline Neuropathy & 2 & 17 \\
\hline Infection & 1 & 1 \\
\hline Hyponatremia & 1 & 1 \\
\hline Intestinal obstruction & 2 & 2 \\
\hline Fever & 3 & 6 \\
\hline Perianal infection & 1 & 1 \\
\hline Abdominal infection & 1 & 1 \\
\hline Operation site wound infection & 1 & 1 \\
\hline AST elevation & 1 & 1 \\
\hline Lumbar pain & & 1 \\
\hline Thrombus & 1 & 1 \\
\hline Mental change & 3 & 3 \\
\hline Proteinuria & 1 & 2 \\
\hline Fatigue & - & 22 \\
\hline Weight loss & - & 4 \\
\hline Weight gain & - & 2 \\
\hline Cough & - & 3 \\
\hline Hypersensitivity & - & 4 \\
\hline Anorexia & - & 15 \\
\hline Diarrhea & - & 6 \\
\hline Constipation & - & 4 \\
\hline Hematochezia & - & 1 \\
\hline Rash & - & 3 \\
\hline Mucositis & - & 8 \\
\hline Alopecia & - & 1 \\
\hline Epistaxis & - & 7 \\
\hline Anemia & - & 1 \\
\hline Thrombocytopenia & - & 7 \\
\hline Total & 34 & 155 \\
\hline
\end{tabular}

AST, aspartate aminotransferase.

at the second follow-up evaluation compared to the first follow-up. Serum PlGF was elevated at the first follow-up evaluation and was still elevated at the second follow-up evaluation. There were no significant changes in sVEGFR2, IL-8, and size, and no significant correlations were observed between the changes in DCE-MRI parameters and plasma CAF values.

The mean tumor size measured at baseline, first follow-up, and second follow-up was $47.0 \pm 22.0 \mathrm{~mm}, 47.0 \pm 21.3 \mathrm{~mm}$, and $47.0 \pm 22.1 \mathrm{~mm}$, respectively. 

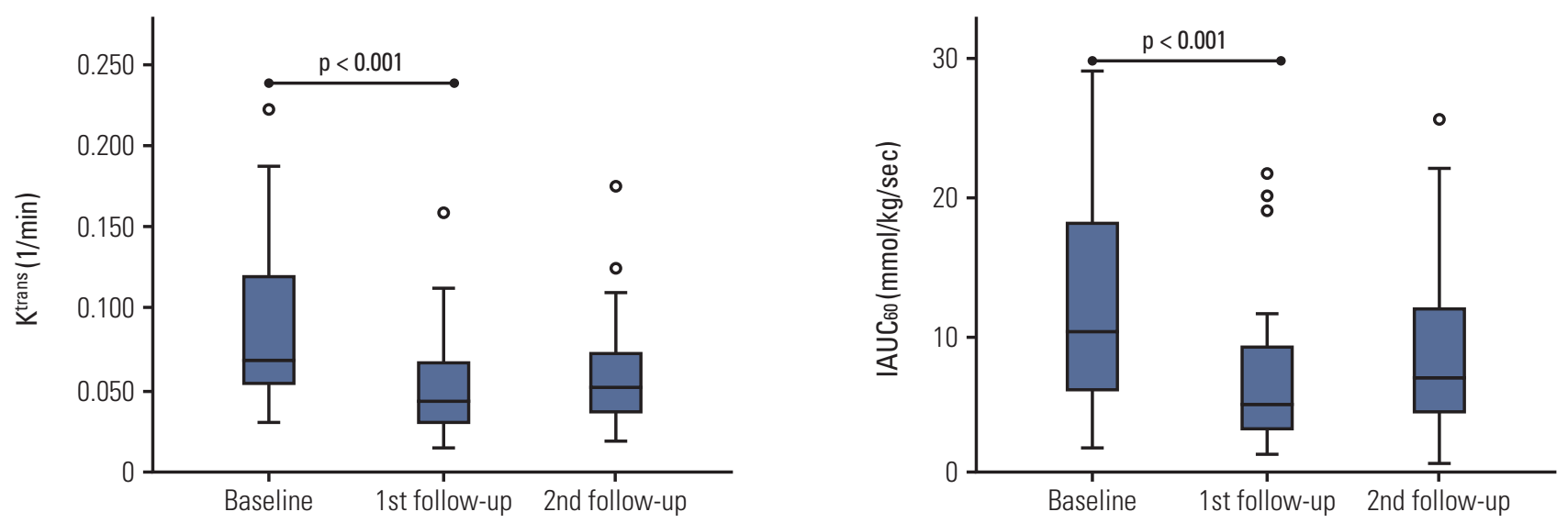

C
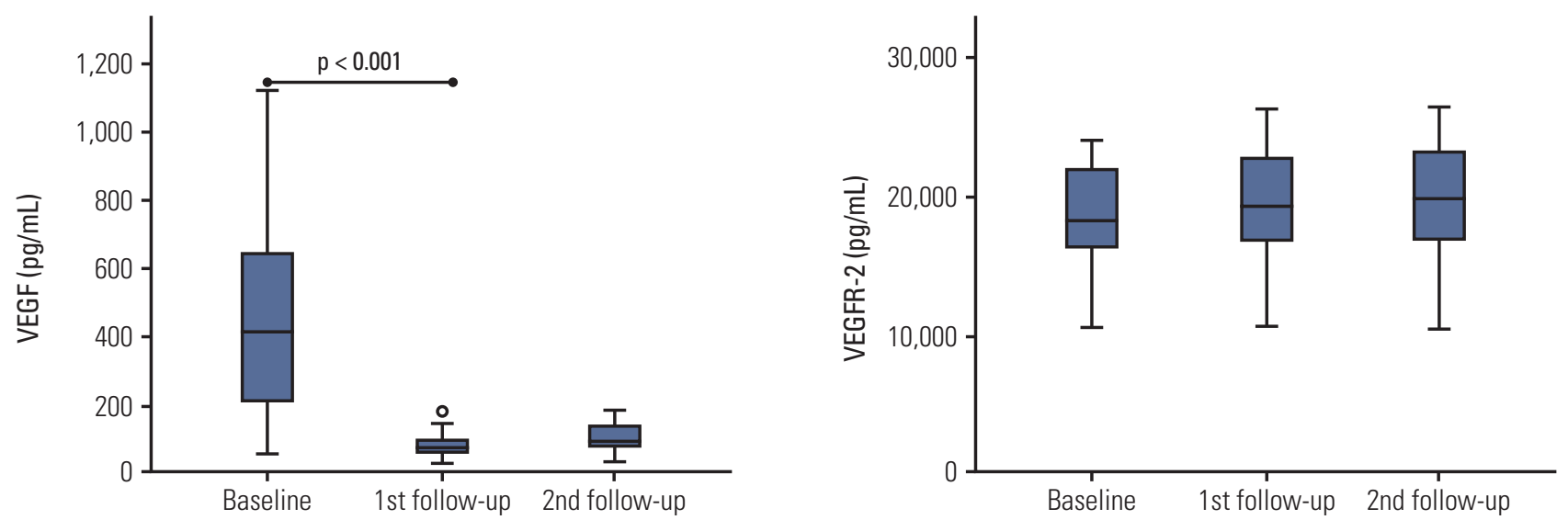

Fig. 2. (A-G) Changes in the values of the dynamic contrast-enhanced magnetic resonance imaging (DCE-MRI) and plasma cytokine and angiogenic factors (CAFs) parameters from baseline, first follow-up after treatment with bevacizumab alone, and second follow-up after treatment with combined therapy. VEGF, vascular endothelial growth factor; VEGFR-2, vascular endothelial growth factor receptor 2; IL-8, interleukin 8; PIGF, placental growth factor. (Continued to the next page)

\section{DCE-MRI parameters and plasma CAFs as prognostic biomarkers}

The results using Kaplan-Meier analysis indicated that PFS time showed correlation with the dichotomized $\mathrm{K}^{\mathrm{trans}}$ (i.e., with the cut-off set at $40 \%$ reduction) variable. $\mathrm{A} \geq 40 \%$ reduction in $\mathrm{K}^{\text {trans }}$ from baseline to 3 days after bevacizumab monotherapy showed significant association with a longer PFS time ( $p=0.025)$. OS time did not show significant associ-

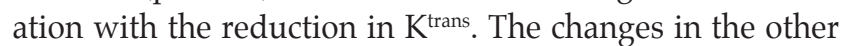
parameters, including IAUC $_{60}$ and plasma CAFs, did not show significant correlation with PFS time (Fig. 3).
When dichotomized at the median values for the baseline DCE-MRI parameters, a high baseline IAUC 60 (cut-off value, 10.337) showed significant association with a longer PFS time $(\mathrm{p}=0.033)$, but not with a longer OS time (Fig. 3). In analysis of the relationships between plasma CAFs and clinical outcomes, there were no associations between baseline serum markers and PFS or OS times.

In multivariate analyses using the Cox regression method, a greater reduction in $\mathrm{K}^{\text {trans }}$ and a high baseline IAUC $_{60}$ were independent predictors for PFS time. Patients with $\mathrm{a} \geq 40 \%$ reduction in $\mathrm{K}^{\text {trans }}$ from baseline to 3 days after bevacizumab monotherapy had a 0.349 times lower hazard of disease pro- 


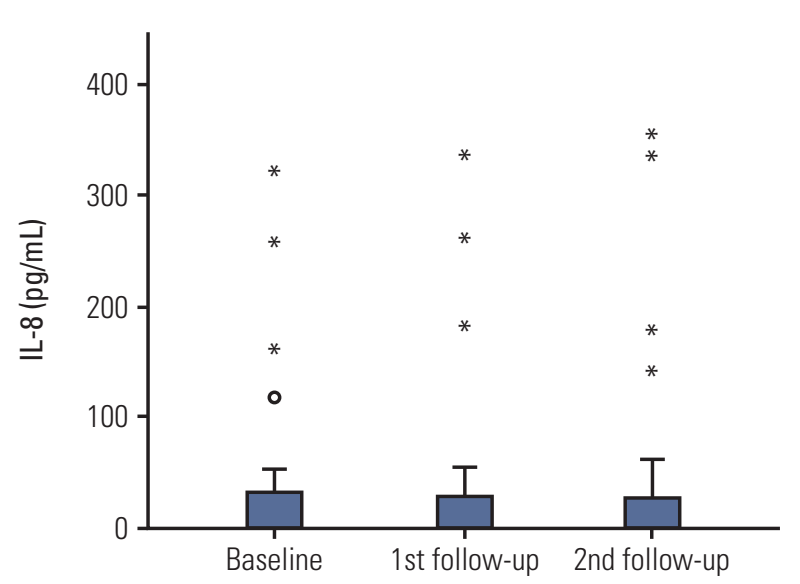

G

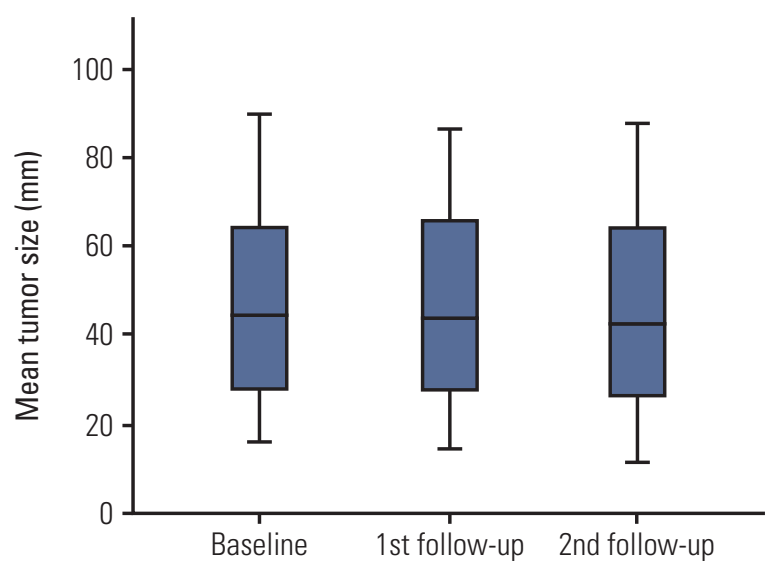

Fig. 2. (Continued from the previous page)

gression (Table 2).

The combined effects of changes in $\mathrm{K}^{\text {trans }}$ and baseline IAUC $_{60}$ were also analyzed. The results using the KaplanMeier method indicated that the patients with both a greater change in $\mathrm{K}^{\text {trans }}$ and a higher baseline IAUC $\mathrm{C}_{60}$ experienced the longest median PFS time (23.8 months, $\mathrm{n}=7$ ); the patients with both the smaller change in $\mathrm{K}^{\text {trans }}$ and the lower baseline IAUC $_{60}$ values had the shortest median PFS times (8.1 months, $n=10, p=0.019$ ). In multivariate analysis, compared with the patients with the greater change in $\mathrm{K}^{\text {trans }}$ and higher baseline IAUC 60 values, the patients with the smaller change in $\mathrm{K}^{\text {trans }}$ and the lower baseline IAUC $_{60}$ values had a six times greater odds of disease progression (hazard ratio [HR], 6.061; 95\% CI, 1.591 to 23.09; $\mathrm{p}=0.008$ ). The differences in OS times were not significantly different (HR, 2.497; $95 \% \mathrm{CI}, 0.643$ to

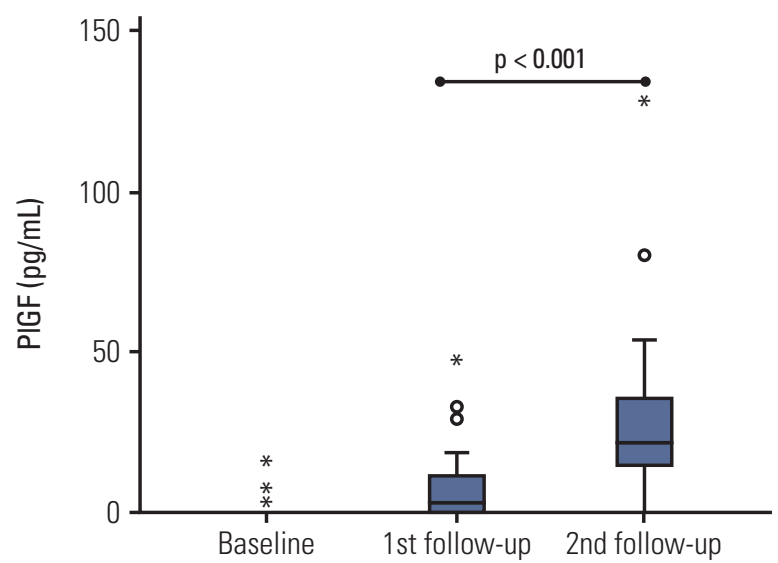


A

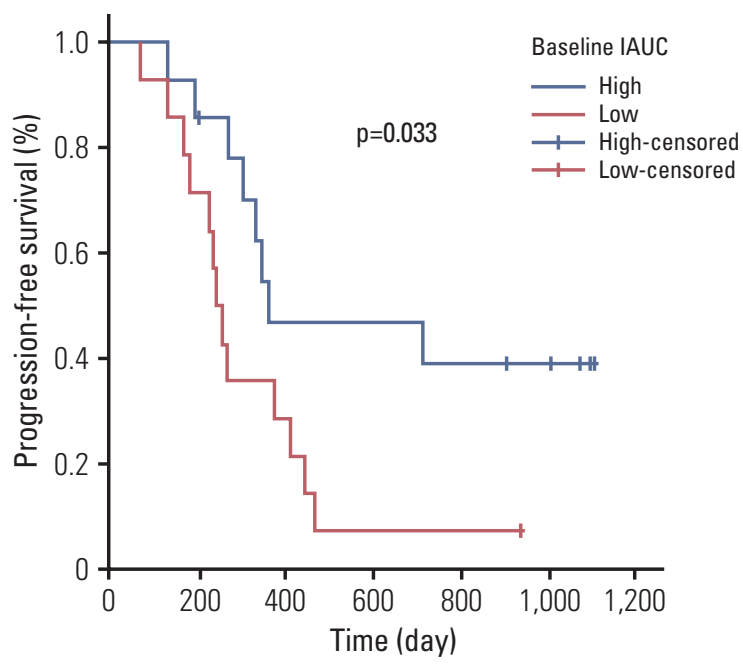

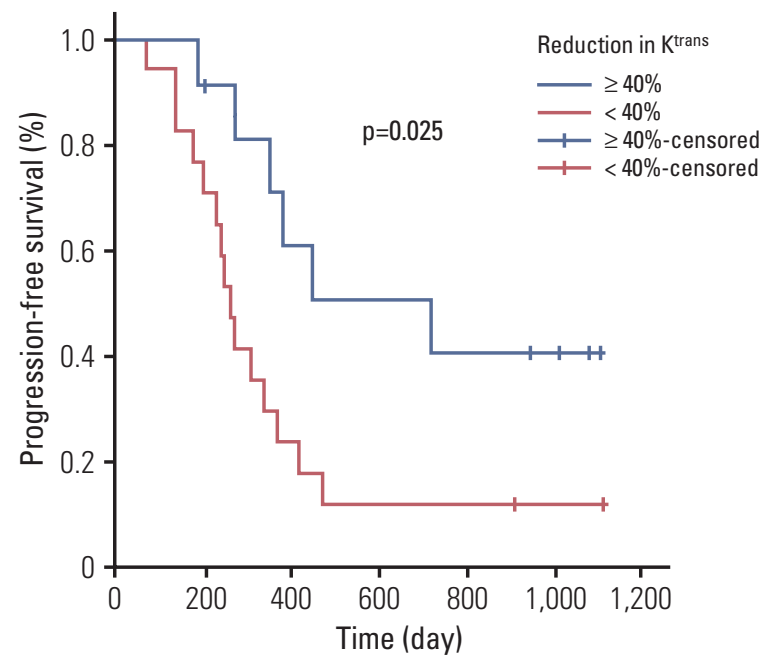

Fig. 3. Kaplan-Meier estimates for progression-free survival time for patients with low and high baseline IAUC60 values (A), and a $\mathrm{K}^{\mathrm{trans}}$ reduction at the first follow-up (B).

\section{Discussion}

The OS time of 22.7 months and the PFS time of 11.2 months obtained in our study compared favorably with the OS time of 21.3 months and the PFS time of 10.4 months obtained in a previous randomized controlled phase III trial using concurrent FOLFOX-6+B regimen [1]. Although our study was limited by the exploratory single-arm trial study design, this result suggested that bevacizumab monotherapy followed by concurrent FOLFOX-6+B regimen is comparable to a concurrent FOLFOX-6+B regimen for treatment of colorectal cancer liver metastasis (CRCLM).

The results from this exploratory trial suggested that the patients with $\geq 40 \%$ reduction in $\mathrm{K}^{\text {trans }}$ by 3 days after bevacizumab monotherapy had a significantly longer PFS time, suggesting that they received a clinical benefit from bevacizumab that those with a $<40 \%$ change in $\mathrm{K}^{\text {trans }}$ did not receive. The results might imply that the prognosis for combined chemotherapy using bevacizumab can be predicted by the microvascular changes induced by bevacizumab alone. The change was detected as early as 3 days after bevacizumab monotherapy, which is much earlier than the size reductions induced by the combined therapy. Therefore, the decision to continue with bevacizumab, or not, can be made as early as 3 days after administration of bevacizumab monotherapy, with minimal cost or side effects. This result was consistent with the results of previous studies reporting that $\mathrm{K}^{\text {trans }}$ can be used as a prognostic biomarker that reflects microvascular change after antiangiogenic therapy, even when the target tumors, the drugs used, or the day that DCEMRI results are obtained (usually 14 days after treatment) are different $[9,11,12,19-21]$. Our study results support the hypothesis that DCE-MRI can be used as a prognostic biomarker for the bevacizumab therapy used for CRCLM treatment.

Based on the results of our study, baseline $\mathrm{IAUC}_{60}$ values can be used as a prognostic biomarker for the outcome of therapy using bevacizumab. This study found that patients with a higher than median value baseline IAUC $_{60}$ had a significantly longer PFS time, suggesting that they could receive clinical benefit from combined therapy using bevacizumab.

The power as a prognostic biomarker is greater when considering the change in $\mathrm{K}^{\text {trans }}$ combined with the baseline IAUC $_{60}$ value, compared with the values considered separately. A patient with $\mathrm{a} \geq 40 \%$ change in $\mathrm{K}^{\text {trans }}$ by 3 days after bevacizumab therapy and a relatively high baseline IAUC 60 should benefit from combined therapy with bevacizumab.

This study found that all of the DCE-MRI parameters ( $\mathrm{K}^{\text {trans }}$ and $\mathrm{IAUC}_{60}$ ) decreased significantly by 3 days after bevacizumab monotherapy, but not after combined chemotherapy (maintaining the lower level as similar to that at 3 days after bevacizumab monotherapy).

These results reflected the fact that perfusion changes in the patients with CRCLM were induced by bevacizumab alone, but not by combined chemotherapy. When used for the treatment of human rectal cancer, the direct effects of bevacizumab on tumor physiology include decreases in 
Table 2. Results of multivariate analyses of the predictors of progression-free survival

\begin{tabular}{lcc} 
Variable & HR $(95 \%$ CI $)$ & p-value \\
K$^{\text {trans }}$ baseline & $0.716(0.301-1.705)$ & 0.451 \\
K$^{\text {trans }} 1$ st follow-up_baseline & $0.349(0.133-0.912)$ & 0.032 \\
\hline IAUC60_baseline & $0.39(0.159-0.957)$ & 0.040 \\
IAUC60 1st follow-up_baseline & $1.014(0.425-2.42)$ & 0.975 \\
VEGF_baseline & $1.217(0.508-2.915)$ & 0.660 \\
VEGF 1st follow-up_baseline & $1.68(0.224-12.589)$ & 0.614 \\
VEGFR-2_baseline & $0.523(0.216-1.268)$ & 0.151 \\
VEGFR-2 1st follow-up_baseline & $4.328(0.912-20.532)$ & 0.065 \\
IL-8_baseline & - & - \\
IL-8 1st follow-up_baseline & $0.613(0.205-1.833)$ & 0.381 \\
PIGF_baseline & - & - \\
PlGF 1st follow-up_baseline & $1.034(0.303-3.53)$ & 0.957 \\
\hline
\end{tabular}

The median baseline values for IL- 8 and PIGF were zero, thus the baseline covariates of those parameters were excluded from the multivariate analyses. The group with the lower baseline value, compared with the median value, was the baseline reference group. The group with a $<40 \%$ change was the reference group for the analysis of the change in values over time. If the change between the first follow-up and the second follow-up was not significant for a parameter, it was excluded from the multivariate analysis. $\mathrm{HR}$, hazard ratio; $\mathrm{CI}$, confidence interval; VEGF, vascular endothelial growth factor; $\mathrm{IL}$, interleukin; PlGF, placental growth factor.

tumor blood perfusion, blood volume, interstitial fluid pressure, and microvessel density [22,23]. These effects are induced by a direct antipermeability effect, vasoconstriction, a direct antiangiogenic effect inhibiting the formation of immature vessels, and / or apoptosis of proliferating endothelial cells [8]. Since the development of antiangiogenic therapy, perfusion imaging has been extensively investigated as a method for use in validation of the biologic efficacy of the molecular target agents on tumor tissue. DCE-MRI, one of the imaging modalities for perfusion imaging, is advantageous for the quantitative measurement of tumor microcirculation in vivo. This approach is noninvasive, repeatable, without radiation hazards, and includes anatomic details of the tumor. Because angiogenesis is a direct or indirect target of many new anticancer drugs, DCE-MRI is an appealing biomarker candidate for assessment of tumor angiogenic activity and for monitoring the effects of antiangiogenic agents [8]. Our study results indicated that DCE-MRI can be used a pharmacodynamic biomarker of bevacizumab therapy of CRCLM.

We also assessed the effects of bevacizumab on plasma CAFs (IL-8, VEGF-A, vascular endothelial growth factor receptor 2 [VEGFR-2], and PIGF) to examine the systemic response. VEGF (also referred to as VEGF-A) has a crucial role in physiological and pathological angiogenesis, and VEGF-targeted agents have been validated as providing clinical benefits in the treatment of malignant disease [22,24]. To better understand the systemic effect of bevacizumab, which is a VEGF-targeted agent, more information about the biology of the VEGF ligand/receptor system is needed [24]. The mammalian VEGF family of ligands consists of five glycoproteins (i.e., VEGF-A [commonly referred to as VEGF], VEGF-B, VEGF-, VEGF-D, and PlGF). The VEGF ligands bind to and activate three different type III receptor tyrosine kinases (known as VEGFR-1, VEGFR-2, and VEGFR-3), which have similar structures. Each ligand of the VEGF family has a distinctive binding specificity for one of the three receptors, therefore a diversity of functions can be mediated. VEGFR-2 is the key mediator of VEGF-induced angiogenesis [24]. Our results showed a significant decrease in circulating VEGF-A by 3 days after bevacizumab monotherapy, but not after combined chemotherapy. Most of the circulating VEGFA was likely bound to bevacizumab as early as 3 days after bevacizumab monotherapy. This result was consistent with our predictions and with other data from blood marker studies of bevacizumab used for the treatment of colorectal cancer and other cancers $[7,25]$.

An increase in plasma PIGF levels was observed by 3 days after bevacizumab monotherapy and a more significant increase by 3 days after combined chemotherapy. Inhibition of VEGF signaling leads to a presumed compensatory increase in expression of other angiogenic factors, resulting in resistance to VEGF-targeted therapy; PIGF levels increase in plasma following blockade of VEGF signaling [24]. The delayed response of PIGF in our study can be explained by the consequential effect of the blockade of VEGF-A with 

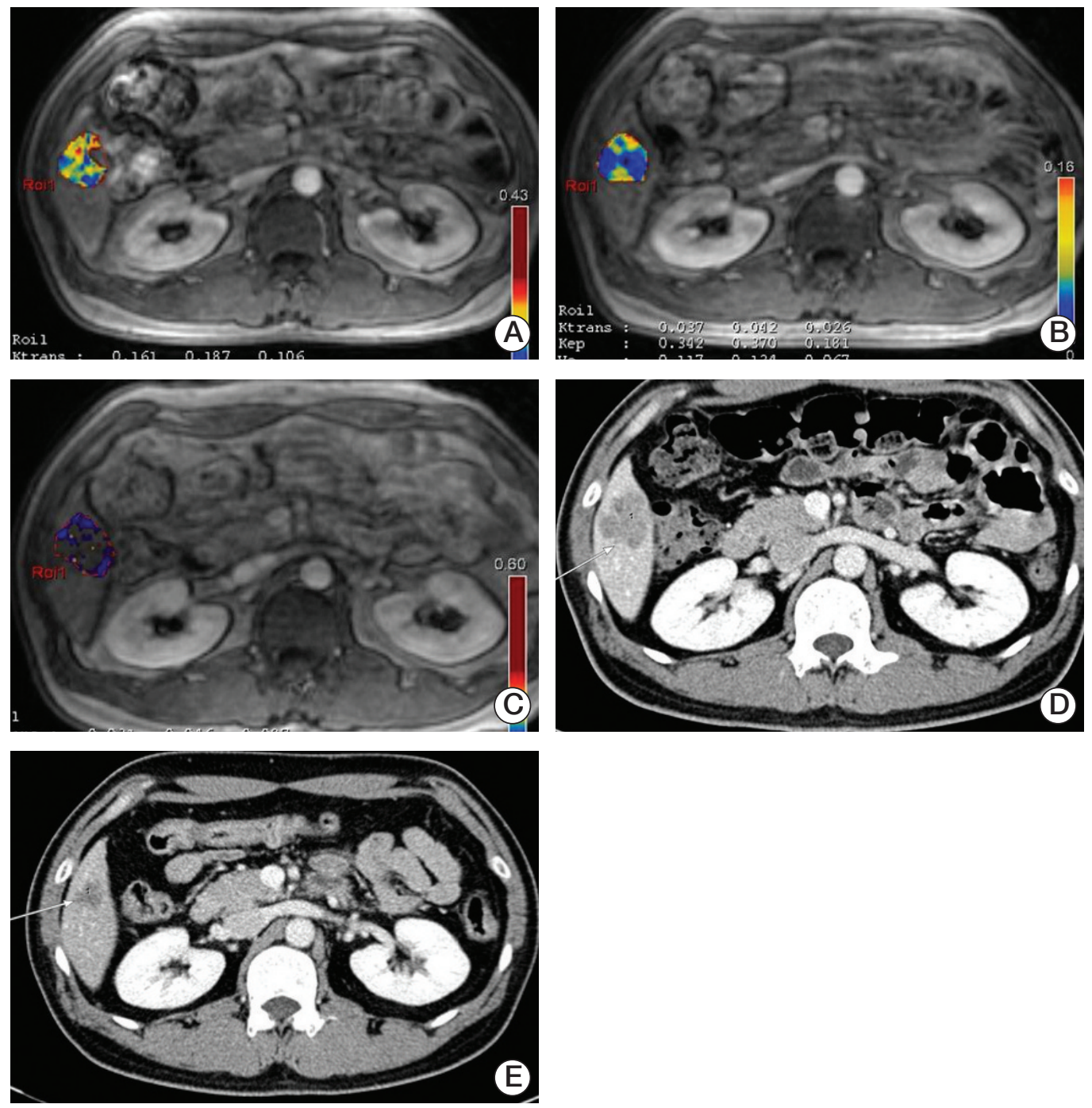

Fig. 4. A 39-year-old male patient had rectal cancer with one hepatic metastatic lesion. (A) On the baseline image the IAUC60 value was 22.29, which was a higher baseline group value. The baseline $K^{\text {trans }}$ value was 0.187 . (B) On the first follow-up image after bevacizumab monotherapy, the patient had a $66 \%$ reduction in $\mathrm{K}^{\text {trans }}(0.037)$. The dynamic contrast enhancedmagnetic resonance imaging results indicated that there were no significant changes in the perfusion parameters between the first and second follow-up (C) (0.031). Based on the large reduction in $\mathrm{K}^{\text {trans }}$, and the high baseline IAUC 60 level, this patient was expected to have a good response to the treatment and to have a good prognosis. Compared with the baseline computed tomography (CT) image (D), the metastatic lesion had decreased in size by the 3-month CT follow-up (E).

bevacizumab.

Kopetz et al. [7] reported that an elevated IL-8 level at baseline is associated with a shorter PFS time, but our study found no association between baseline IL-8 values and PFS time. This result could be due to the different time points used for plasma sampling, which were 3 days after treatment in our study and before progression in their study (median PFS time, 12.8 months). Circulating cytokine levels may reflect the responses of non-neoplastic host tissue and of neoplastic tumor tissue to treatment [7]. The associations between plasma angiogenic factors and clinical outcomes remain unclear, probably due to the complexities of tumor angiogenesis, angiogenic factors, and resistance mechanisms to the anti-angiogenic therapy $[6,7,24]$.

This study had the limitation of small sample size. Therefore, the results of our study, usefulness of $K^{\text {trans }}$ change as a 
prognostic biomarker, cannot be directly applied to the daily practice. Further study with a larger sample size is needed.

\section{Conflicts of Interest}

Conflict of interest relevant to this article was not reported.

\section{Conclusion}

In conclusion, DCE-MRI parameters and CAFs are pharmacodynamic biomarkers of bevacizumab for CRCLM. In our study, change in $\mathrm{K}^{\text {trans }}$ at 3 days after bevacizumab monotherapy was a favorable prognostic factor, but the value of CAFs as a prognostic biomarker was not found.

\section{Acknowledgments}

This study was supported by a grant from the National Research Foundation of Korea (7-2010-0288).

\section{References}

1. Saltz LB, Clarke S, Diaz-Rubio E, Scheithauer W, Figer A, Wong $R$, et al. Bevacizumab in combination with oxaliplatinbased chemotherapy as first-line therapy in metastatic colorectal cancer: a randomized phase III study. J Clin Oncol. 2008;26: 2013-9.

2. Meyerhardt JA, Li L, Sanoff HK, Carpenter W 4th, Schrag D. Effectiveness of bevacizumab with first-line combination chemotherapy for Medicare patients with stage IV colorectal cancer. J Clin Oncol. 2012;30:608-15.

3. Ducreux M, Adenis A, Pignon JP, Francois E, Chauffert B, Ichante JL, et al. Efficacy and safety of bevacizumab-based combination regimens in patients with previously untreated metastatic colorectal cancer: final results from a randomised phase II study of bevacizumab plus 5-fluorouracil, leucovorin plus irinotecan versus bevacizumab plus capecitabine plus irinotecan (FNCLCC ACCORD 13/ 0503 study). Eur J Cancer. 2013;49:1236-45.

4. Jain RK. Normalization of tumor vasculature: an emerging concept in antiangiogenic therapy. Science. 2005;307:58-62.

5. Hegde PS, Jubb AM, Chen D, Li NF, Meng YG, Bernaards C, et al. Predictive impact of circulating vascular endothelial growth factor in four phase III trials evaluating bevacizumab. Clin Cancer Res. 2013;19:929-37.

6. Jain RK, Duda DG, Willett CG, Sahani DV, Zhu AX, Loeffler JS, et al. Biomarkers of response and resistance to antiangiogenic therapy. Nat Rev Clin Oncol. 2009;6:327-38.

7. Kopetz S, Hoff PM, Morris JS, Wolff RA, Eng C, Glover KY, et al. Phase II trial of infusional fluorouracil, irinotecan, and bevacizumab for metastatic colorectal cancer: efficacy and circulating angiogenic biomarkers associated with therapeutic resistance. J Clin Oncol. 2010;28:453-9.

8. O'Connor JP, Jackson A, Parker GJ, Roberts C, Jayson GC. Dynamic contrast-enhanced MRI in clinical trials of antivascular therapies. Nat Rev Clin Oncol. 2012;9:167-77.

9. Wedam SB, Low JA, Yang SX, Chow CK, Choyke P, Danforth
D, et al. Antiangiogenic and antitumor effects of bevacizumab in patients with inflammatory and locally advanced breast cancer. J Clin Oncol. 2006;24:769-77.

10. O'Connor JP, Rose CJ, Jackson A, Watson Y, Cheung S, Maders F, et al. DCE-MRI biomarkers of tumour heterogeneity predict CRC liver metastasis shrinkage following bevacizumab and FOLFOX-6. Br J Cancer. 2011;105:139-45.

11. De Bruyne S, Van Damme N, Smeets P, Ferdinande L, Ceelen W, Mertens J, et al. Value of DCE-MRI and FDG-PET/CT in the prediction of response to preoperative chemotherapy with bevacizumab for colorectal liver metastases. Br J Cancer. 2012; 106:1926-33.

12. Drevs J, Siegert $P$, Medinger M, Mross K, Strecker R, Zirrgiebel U, et al. Phase I clinical study of AZD2171, an oral vascular endothelial growth factor signaling inhibitor, in patients with advanced solid tumors. J Clin Oncol. 2007;25:3045-54.

13. Morgan B, Thomas AL, Drevs J, Hennig J, Buchert M, Jivan A, et al. Dynamic contrast-enhanced magnetic resonance imaging as a biomarker for the pharmacological response of PTK787/ ZK 222584, an inhibitor of the vascular endothelial growth factor receptor tyrosine kinases, in patients with advanced colorectal cancer and liver metastases: results from two phase I studies. J Clin Oncol. 2003;21:3955-64.

14. Flaherty KT, Rosen MA, Heitjan DF, Gallagher ML, Schwartz $\mathrm{B}$, Schnall MD, et al. Pilot study of DCE-MRI to predict progression-free survival with sorafenib therapy in renal cell carcinoma. Cancer Biol Ther. 2008;7:496-501.

15. Hsu CY, Shen YC, Yu CW, Hsu C, Hu FC, Hsu CH, et al. Dynamic contrast-enhanced magnetic resonance imaging biomarkers predict survival and response in hepatocellular carcinoma patients treated with sorafenib and metronomic tegafur/ uracil. J Hepatol. 2011;55:858-65.

16. Hahn OM, Yang C, Medved M, Karczmar G, Kistner E, Karrison $\mathrm{T}$, et al. Dynamic contrast-enhanced magnetic resonance imaging pharmacodynamic biomarker study of sorafenib in 
metastatic renal carcinoma. J Clin Oncol. 2008;26:4572-8.

17. Eisenhauer EA, Therasse P, Bogaerts J, Schwartz LH, Sargent D, Ford R, et al. New response evaluation criteria in solid tumours: revised RECIST guideline (version 1.1). Eur J Cancer. 2009;45:228-47.

18. Chefd'Hotel C, Hermosillo G, Faugeras O. Flows of diffeomorphisms for multimodal image registration. In: Proceedings of the 2002 IEEE International Symposium on Biomedical Imaging; 2002 Jun 7-10; Washington, DC, USA. New York: IEEE; 2002. p. 753-6.

19. Evelhoch JL. Key factors in the acquisition of contrast kinetic data for oncology. J Magn Reson Imaging. 1999;10:254-9.

20. Zhu AX, Sahani DV, Duda DG, di Tomaso E, Ancukiewicz M, Catalano OA, et al. Efficacy, safety, and potential biomarkers of sunitinib monotherapy in advanced hepatocellular carcinoma: a phase II study. J Clin Oncol. 2009;27:3027-35.

21. Port RE, Bernstein LJ, Barboriak DP, Xu L, Roberts TP, van
Bruggen N. Noncompartmental kinetic analysis of DCE-MRI data from malignant tumors: Application to glioblastoma treated with bevacizumab. Magn Reson Med. 2010;64:408-17.

22. Willett CG, Boucher Y, di Tomaso E, Duda DG, Munn LL, Tong RT, et al. Direct evidence that the VEGF-specific antibody bevacizumab has antivascular effects in human rectal cancer. Nat Med. 2004;10:145-7.

23. Ferrara N, Gerber HP, LeCouter J. The biology of VEGF and its receptors. Nat Med. 2003;9:669-76.

24. Ellis LM, Hicklin DJ. Pathways mediating resistance to vascular endothelial growth factor-targeted therapy. Clin Cancer Res. 2008;14:6371-5.

25. Loupakis F, Cremolini C, Fioravanti A, Orlandi P, Salvatore L, Masi G, et al. Pharmacodynamic and pharmacogenetic angiogenesis-related markers of first-line FOLFOXIRI plus bevacizumab schedule in metastatic colorectal cancer. Br J Cancer. 2011;104:1262-9. 